EPJ Web of Conferences 114,02151 (2016)

DOI: $10.1051 /$ epjconf/201611402151

(C) Owned by the authors, published by EDP Sciences, 2016

\title{
Mathematical modeling of control system for the experimental steam generator
}

\author{
Szymon Podlasek ${ }^{1}$, Krzysztof Lalik ${ }^{2}$, Mariusz Filipowicz ${ }^{1}, K_{\text {rzysztof Sornek }}^{1, a}$, Robert Kupski ${ }^{3}$ and Anita Raś ${ }^{3}$ \\ ${ }^{1}$ A GH University, Faculty of Energy and Fuels, Mickiewicza Av. 30, 30-059 Krakow, Poland \\ ${ }^{2}$ AGH University, Faculty of Mechanical Engineering and Robotics, Mickiewicza Av. 30, 30-059 Krakow, Poland \\ ${ }^{3}$ Dom Project Sp. z o.o., ul. Willowa 19, 32-085 Modlniczka, Poland
}

\begin{abstract}
A steam generator is an essential unit of each cogeneration system using steam machines. Currently one of the cheapest ways of the steam generation can be application of old steam generators came from army surplus store. They have relatively simple construction and in case of not so exploited units - quite good general conditions, and functionality of mechanical components. By contrast, electrical components and control systems (mostly based on relay automatics) are definitely obsolete. It is not possible to use such units with cooperation of steam bus or with steam engines. In particular, there is no possibility for automatically adjustment of the pressure and the temperature of the generated steam supplying steam engines. Such adjustment is necessary in case of variation of a generator load. The paper is devoted to description of improvement of an exemplary unit together with construction of the measurement-control system based on a PLC. The aim was to enable for communication between the steam generator and controllers of the steam bus and steam engines in order to construction of a complete, fully autonomic and maintenance-free microcogeneration system.
\end{abstract}

\section{Introduction}

According to the new trends, traditional heat sources are going to be replaced by combined heat and power systems (CHP). Such units integrate the production of heat and electricity in one highly efficient technology process. Up to now, cogeneration systems were used almost only in the professional power industry, but nowadays, there are more and more popular also in distributed energy solutions. Due to installed power, distributed energy systems can be divided as follows:

- Micro scale systems - up to $50 \mathrm{kWe}$,

- Small scale systems - from $50 \mathrm{kWe}$ to $1 \mathrm{MWe}$,

- Medium scale systems - from $1 \mathrm{MWe}$ to $50 \mathrm{MWe}$,

- Large scale systems - from $50 \mathrm{MWe}$ to $150 \mathrm{MWe}$.

Currently available microcogeneration devices use several different technologies, including internal and external combustion engines (Stirling engines, Otto engines etc.), steam expansion engines and fuel cells. Many of developed solutions are still in the research and application stage and only a small part of them will be commercially available in the future. Such units are mainly fired by natural gas or oil. On the other hand, if we take into account steam turbines and steam engines (devices suitable to cooperate with external heat sources), we can use biomass fired boilers as heat sources. The huge potential is connected with the use of configured in such way CHP units [1].

The operation of steam units is based on the Rankine Cycle (the same cycle, which is used in the professional power plants). The main part of such systems is a steam generator (e.g. a steam boiler). Heat generated during the combustion process allows to evaporate water, which is used as a working medium. Steam goes from boiler to the turbine and expands passing the blades disposed on the rotor (transfers to the rotor the part of its energy). Mechanical energy is converted to the power using a dedicated power generator. In the typical power plant, partially expanded medium goes next to the condenser what allows to utilize the heat of the condensation.

An extension of classical Rankine cycle is the Organic Rankine cycle (ORC). In ORC cycle the working medium (water) is replaced by low-boiling fluid, such as silicone oil, benzene, toluene etc. The use of organic working fluids results in the ORC cycle having several advantages over the steam cycle. Higher molecular weight than water increases the mass flow rate of the fluid for the same size of turbine. The result is a better power generation efficiency (about 10-20\%). Most importantly, the boiling point of ORC fluids is much lower than that of water, so they can be applied in lower temperatures [2].

\footnotetext{
${ }^{\mathrm{a}}$ Corresponding author: ksornek@agh.edu.pl
} 
The idea of a small cogeneration system based on the use of steam turbines (or steam engines) was shown in figure. 1. The comparison of classical steam cycle and ORC cycle was shown in the T-S diagram in figure. 2 .

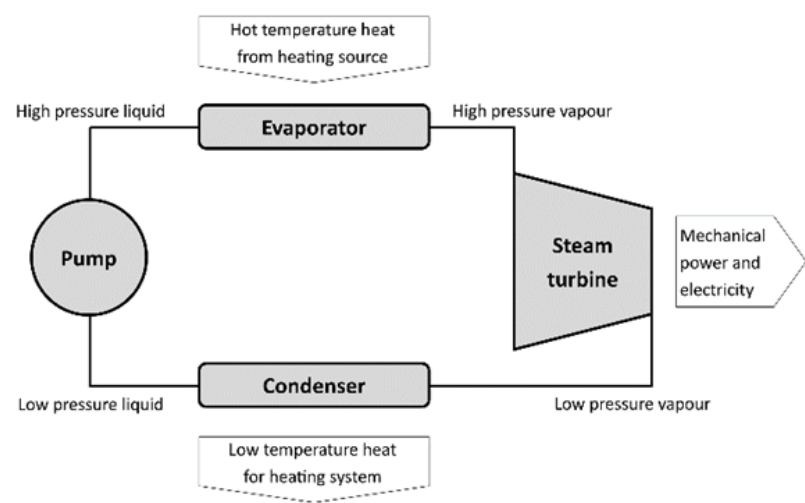

Figure 1. The idea of a small cogeneration system (source: [2]).
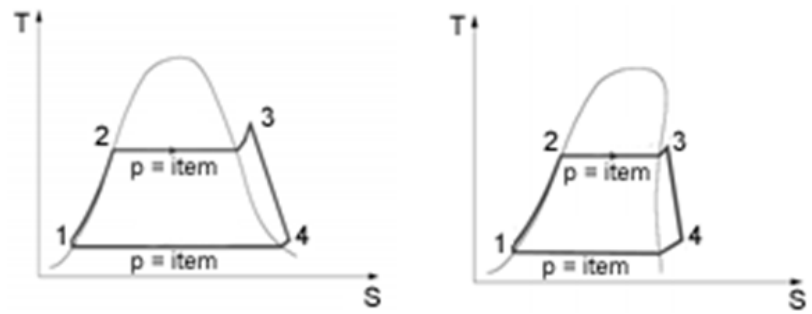

Figure 2. Comparison of classic steam and ORC cycle [3].

\section{Biomass boilers as a heat sources for CHP systems}

According to assumptions presented in the EU Energy, Transport and GHG Emission Trends to 2050 (document published by the European Commission), the overall share of renewable energy sources in the electricity production will achieve a level of $50 \%$ in 2050 [4]. The increase in electricity production from RES will be connected with the dynamic development of distributed energy systems. Power will be generated e.g. in classical Rankine Cycle and Organic Rankine Cycle based systems powered by biomass-fired boilers. The expected share of biomass in local CHP systems and network heating up to 2050 was presented in figure 3 .

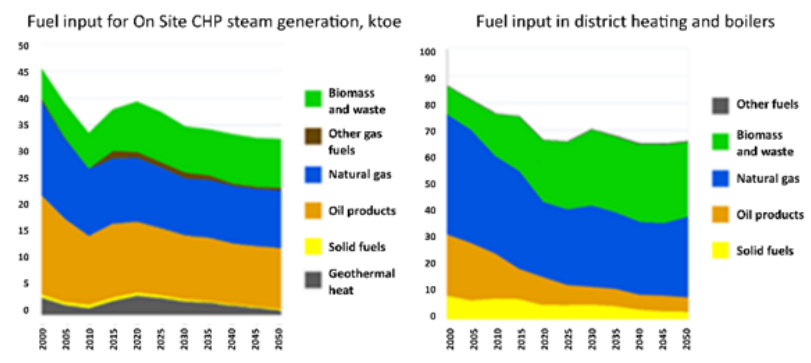

Figure 3. Predicted biomass share in local CHP systems and heating systems (own work based on [4]).

The use of cogeneration systems equipped with the biomass-fired boilers is possible both in the case of residential buildings, public buildings and farms [5]. It is really important especially in the cases, where the problems with access to the grid occur. In such facilities the oil-fired external combustion engines, which operation is really expensive are mostly used. Replacing such devices by biomass-fired CHP systems will allow reducing energy costs and obtaining full or partial energy independence.

\section{Testing version of the cogeneration system}

All tests of the steam generator were done using simple set-up shown in figure 4. The installation includes steam engines and steam turbine with power of range 1-10 kW. Some auxiliary elements are also indicated.

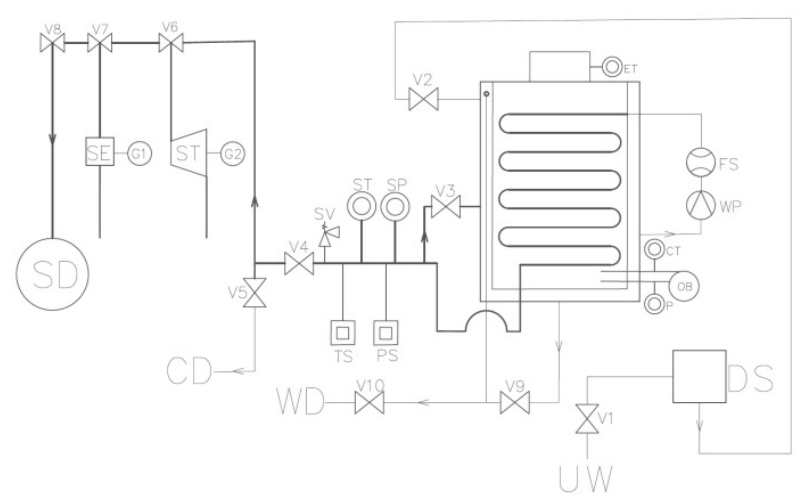

Figure 4. Diagram of simple version of the cogeneration system including steam machines, steam bus and steam generator. Description of symbols in the text.

The meaning of symbols in figure 4 is the following:

V - Ball Vavle

SV - Safety Vavle

UW - Untreated Water

DS - Demineralizing Station

ET - Exhaust Temperature

FS - Flow Sensor

WP - Water Pump

CT - Control Temperature

P - Photoresistor

WD - Water Discharge

ST - Steam Temperature

SP - Steam Pressure

TS - Temperature Switch

PS - Pressure Switch

CD - Condensate Discharge

SD - Steam Discharge

SE - Steam Engine

ST - Stem Turbine

G - Generator.

It is visible that the steam machines are mounted on the common steam bus and each engine is equipped with a cut-off valve. Also, the cut-off valves are mounted between the steam generators and the steam bus.

In the considered system certain parameters of working medium in the close loop have to be secured. Application of some number of the auxiliary elements 
such as dehumidifiers, separators, reduction valves etc. influence on proper operation of the system and allows to obtain steam with given parameters, allowing then for safe and efficient operation of the engines. Those factors also may reduce possibility of mechanical damages in the whole system. Very efficient control system cooperating with the other elements allows for generation steam with constant parameters of pressure and temperature. It increases security of the system operations and also increase period of the continuous unit operation.

In the installation, role of actuators perform the steam machines and the microturbine. This complete steam system will enable to obtain characteristics of the installed machines in the operational conditions.

The unit made in 1988 and modernized in 2015 was used as a steam generator. The nominal power is ca. $130 \mathrm{~kW}_{\mathrm{t}}$ and steam output - ca. $180 \mathrm{~kg} / \mathrm{h}$. It is example of flow water-tube boiler. Heating and evaporation of the medium takes place in the coil directly heated by the fuel flame. A small volume of the water/steam inside the coil makes some difficultness regarding securing constant parameters of the generated stem. Hence, advanced control system of burner is required to eliminate this disadvantage. The fuel and air supply to the burner should be strictly controlled. In the considered unit the fuel pump and the fan are placed on the common shaft, what makes that these elements are dependent. It means that only rotational speed of the shaft can be used to control the burner power. Unfortunately, the measurements of the temperature directly in the coil are impossible. In this way some delays in the system have to be taken into account in a control algorithm.

Visualization of the steam generator is shown in figure 5.

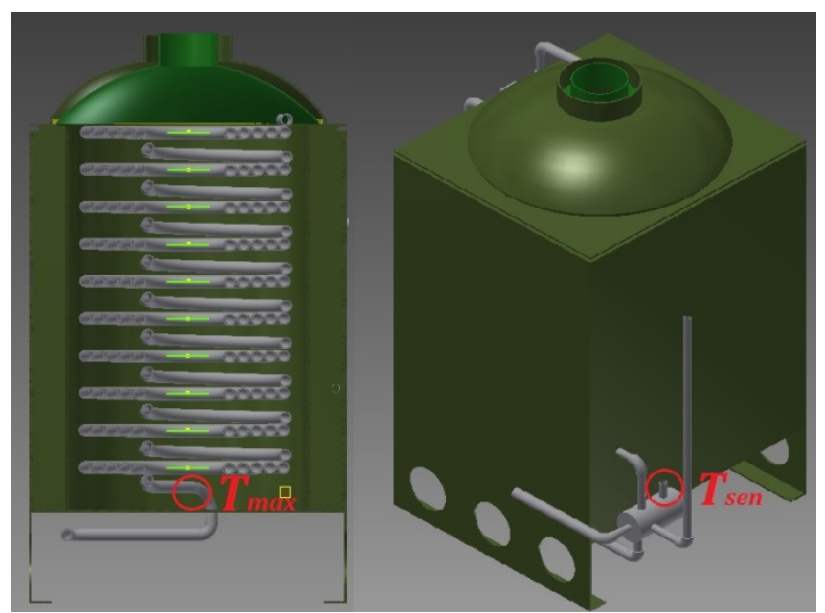

Figure 5. The steam generator - outlook view (left) nd the cross section (right). Place of the maximal temperature $T_{\max }$ and measuring point $\mathrm{T}_{\mathrm{sen}}$ are indicated.

It is visible that the steam temperature sensor is placed in some distance from point of maximal temperature. The photo of the coil is presented in figure 6.

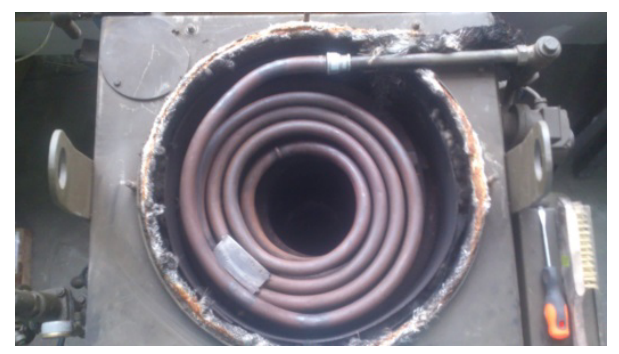

Figure 6. Upper view of the coil, the upper cover with smoke conduit is removed. The water volume of the coil is ca. 30 liters.

The biggest part of the generator is the combustion chamber made of two layers of the boiler steel separated by the insulation layer. Inside the combustion chamber the coil is placed. The water jacket prevents the generator overheating and initially heats the water. Before filling the water jacked, the municipal water has to be treated to prevent appearance of limescale, and then is directed to the water jacket. The formation of limescale has to be reduced because, due to the small diameter of the coil tube, the water flow may be strongly limited or even stopped. Also the heat transfer from the flame to the coil is decreased. The water is sucked from the water jacket by positive displacement pump and flows through the filter to the coil. During its flow, the water is heated and evaporation took place. The set of manual valves allows for initial circulation of the water inside the steam generator, and after reaching required parameters, the steam is directed to the steam bus.

In this way, the coil in such arrangement plays triple role: heater, evaporator and superheater. Due to volume extension of the working medium, the diameter of the coil tube is larger in the "steam part".

The operation of a typical old steam generator with the original automatic do not allow for stable properties of the generated steam. Strong pressure and temperature fluctuations unfortunately exist.

To solve this problem, a dedicated PLC (programmable logic controller) and software is required. Description of this controller, its configuration and tuning and some results of initial test are discussed in the further part of the paper.

\section{Construction of dedicated control system}

An electrical control circuit was updated during modernization of the steam generator. Most of the actuators and sensors were replaced. The outlook diagram of the measurement stand is presented in figure 7. The variation of the ventilator's rotational velocity results in variation of air amount supplied to the combustion chamber. The shaft of the ventilator is coupled by a flexible clutch with the shaft of the fuel pomp. It results also with variation of the fuel dose delivered to the combustion chamber. Therefore, the thermal power of the generator is strictly correlated with the speed of the ventilator. 


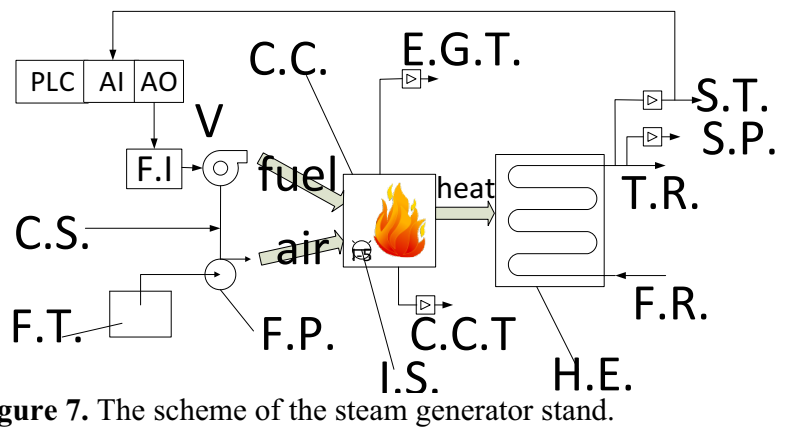

The meaning of symbols in figure 7 is the following:

C.C. - combustion chamber

C.C.T - combustion chamber temperature

C.S. - coupled shafts

E.G.T. - exhaust gas temperature

F.I. - frequency inverter

F.P. - fuel pomp

F.R. - from receiver

F.T. - fuel tank

H.E. - heat exchanger

I.S. - ignition system

S.P. - steam pressure

S.T. - steam temperature

T.R. - to receiver

V. - ventilator.

The diagram shown in figure 8 presents the structure of the control system. The heating coil temperature sensor is used as a feedback signal and is connected to the analog input module in the PLC. The value of this temperature is subtracted from the setpoint, which is entered from graphical user interface. This calculated error is subsequently transformed by the microprocessor and the appropriate control signal is obtained from its value. This signal is afterwards physically generated from the analog output module and directed to the frequency inverter. The inverter regulates the speed of the ventilator according to this calculated control value. With the variation of the ventilator speed, the amount of the energy generated in the combustion chamber and temperature of working medium vary.

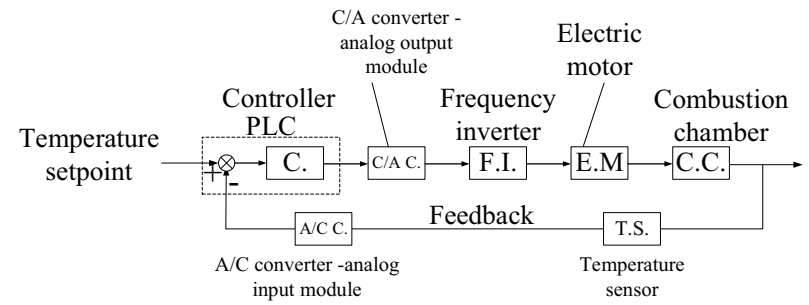

Figure 8. Schematic structure of the steam generator regulation system.

\subsection{Structure of regulatory system}

In systems with large time constant value, like objects such as heating systems, even the simplest type of the control system, like relay control can be applied $[6,7]$. However, in this case these solutions were not applied. This is mainly due to the possibility of control the speed of the asynchronous motor continuously in the entire velocity range by using frequency inverter. The second reason is the highly nonstationariness of the system. Object parameters variability occurs from previously discussed modes of operation of the steam generator and various energy retrieve from it [8]. On the one hand, setting the wide range set of the hysteresis control will occur in oscillations of the process variable around the set point, but the amplitude of those oscillations may be unacceptable by the expected parameters of the steam generator operation. On the other hand, if the range of settings of the hysteresis control loop would be to narrow, then it would be causing an excessive frequent switching of the actuator, which will reduce its lifespan. Additionally, for certain set of control operating parameters, this relay control method may result in rise of the self-excited oscillations and lead to loss of the stability of the process [9].

For these reasons, it was decided to realize the control of the system via a classical PID controller in the form given by the equation 1 .

$$
m(t)=K_{P} e(t)+K_{I} \int_{0}^{t} e(t) d t+K_{D} \frac{d e(t)}{d t},
$$

where $m(t)$ is an control signal and also the controller output, $\mathrm{e}(\mathrm{t})$ is an error signal, $\mathrm{K}_{\mathrm{P}}$ is a proportional gain, $\mathrm{K}_{\mathrm{I}}$ is an integral gain, $a K_{D}$ is a derivative gain.

\subsection{Numerical model}

In order to obtain the most optimal tuning parameters, the offline methods were selected. This choice is made because the most popular method used to find the tuned PID parameters, which is the classic Ziegler-Nichols method, requires from object under examination to be on its stability limit. It might lead even to the destruction of the system.

The offline method requires from designer of the control system a knowledge about the structure of the system. Therefore, an identification experiment was carried out. It involves an examination of the system step response. For this purpose, the open system, where the feedback from the temperature of the collector was disconnected, was tested. The system was stimulated by setting the ventilator motor at $65 \%$ of its rated speed. Then both the motor speed and the temperature of the collector were measured. The experimental results of the identification are shown in figure 9. 


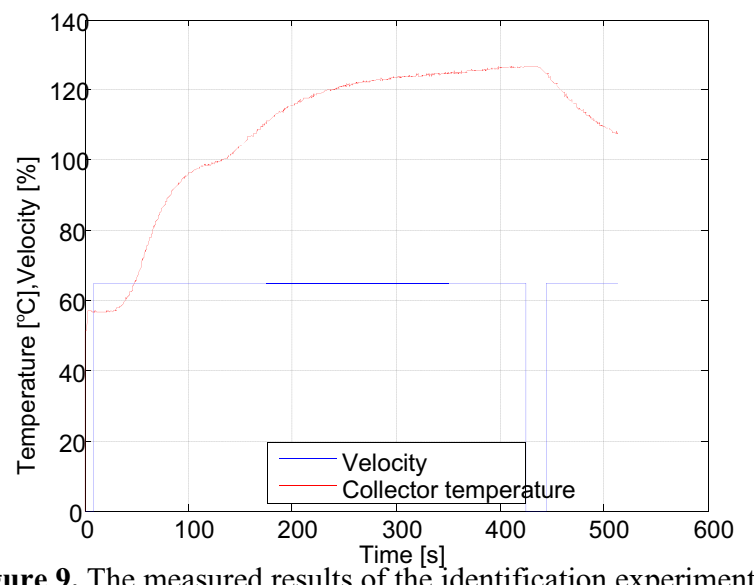

Figure 9. The measured results of the identification experiment

Some trends could be noticed by analyzing the charts showed in figure 9. Firstly, for both kind of medium: the water and the steam, a delay between the reference signal and the system response exists. It occurs because the temperature sensor is located not directly in the heat exchanger, but a little bit behind it, inside the steam collector. Therefore, a classical transport delay is present in the system. This statement is important for building a numerical model of the generator, because such a delay must not be ignored.

The second conclusion, which comes from analysis of the charts showed in figure 9, is the existence of a distinct point of inflection for the temperature of $100^{\circ} \mathrm{C}$. This point is a phase change of the medium from its liquid to gas state. This phenomenon causes a large nonstationarity of the system. However, given the fact that the operating point of the system is above this value, the designer of the control system can build the mathematical model of the system regardless the lower temperatures.

The step response above the inflection point suggests, that the system is an inertial object, like most thermal systems. However, the row of this inertia is still unknown. Therefore, the Nyquist characteristics will be used to investigate its value. According to the control theory, the row of inertia is equal to the number of quarters, through which its spectral characteristics is crossing. The first step to obtain this characteristic is a transition from time domain to frequency domain using power spectral density function $\mathrm{S}$ given by equation 2 .

$$
S(\omega)=\int_{-\infty}^{\infty} R(\tau) e^{-i \omega \tau} d \tau
$$

where $\omega$ is an angular frequency of the signal, $\tau$ is time, and $R(\tau)$ is an signal autocorrelation function.

For input signal $\mathrm{x}$ and system step response $\mathrm{y}$, the spectral transmittance $G(j \omega)$ is given by equation 3 .

$$
G(j \omega)=\frac{s_{x y}}{s_{x x}}
$$

where $S_{x y}$ is a power cross-spectral density function for input and output signal, and $S_{x x}$ a power cross-spectral density function for input signal.

The spectral transmittance of the object obtained using this method is shown in figure 10 .

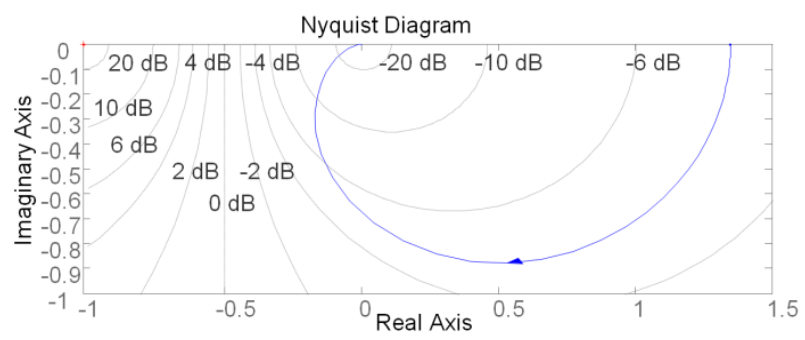

Figure 10. The spectral transmittance of the steam generator obtained as a result of the identification experiment

As it is shown in figure 10, the Nyquist characteristic is present both in the left and right half of the complex plane in quadrant III and IV. Thus, the row of the system inertia may be defined as the second. Considering also the previously described delay in the structure, the entire system of the steam generator may therefore be described as inertial object of the second row with transport delay.

Knowing the structure of the system, the designer can therefore begin to determine the parameters of the system. For this purpose, an iterative method of minimizing the mean square error of the predicted output can be used. This method uses an optimization to minimize the cost function $\mathrm{VN}$, which is given by equation 4 .

$$
V_{N}=\sum_{t=1}^{N} e^{2}(t)
$$

where $\mathrm{e}(\mathrm{t})$ is the difference between the measured output and the predicted model output.

The parameters of the object model were calculated using Matlab ${ }^{\circledR}$ package. Its transfer function $\mathrm{G}(\mathrm{s})$ is given by equation 5 . According to the trends presented in figure 11 , the model response reaches $94.74 \%$ fit to the real measurement stand. It is therefore sufficient to the task of fine-tuning of the PID controller in the offline conditions.

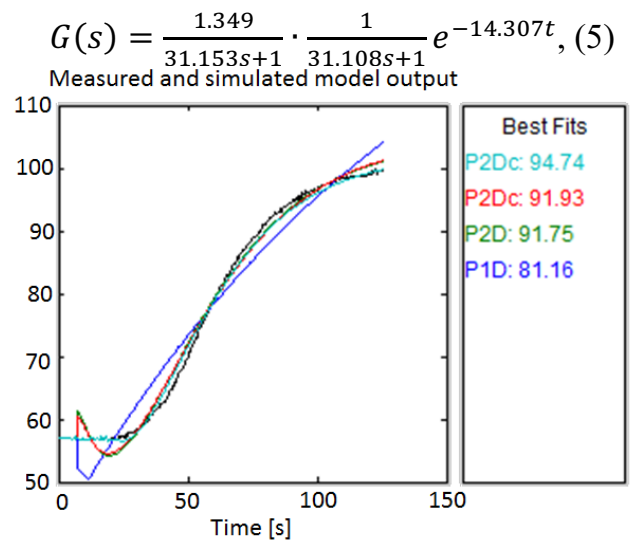

Figure 11. The fit between the model and the measured response. 


\subsection{Controller fine-tuning}

The numerical model in Matlab Simulink ${ }^{\circledR}$ was built using mathematical model of the steam generator given by equation 5 . This model is shown in figure 12 . The signal Constraint toolbox was used to obtain the tuned parameters of the controller. It is using again the algorithm, which minimalize the cost function to obtain the optimum controller settings at the system operating point.

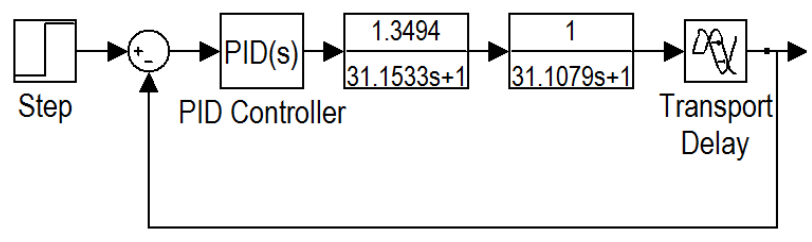

Figure 12. Numerical model of the steam generator with the PID controller.

The setpoint value was determined as $130^{\circ} \mathrm{C}$. For this operating point and presented model the obtained PID controller parameters are shown in Table 1.

Table 1. Optimal PID parameters for operating point $\mathrm{T}=130 \mathrm{C}$.

\begin{tabular}{|l|l|}
\hline Controller Part & Value \\
\hline Proportional Gain $\mathrm{K}_{\mathrm{P}}$ & 1.27 \\
\hline Integral gain $\mathrm{K}_{\mathrm{I}}$ & 0.02 \\
\hline Derivative gain $\mathrm{K}_{\mathrm{D}}$ & 61.99 \\
\hline
\end{tabular}

Taking into account the fact, that the object is highly non-stationary, the parameter $\mathrm{K}_{\mathrm{I}}$ was reduced to value 0.01 . This would reduce the overshoot of the process in other operation point with different temperature.

The biggest impact on the stationarity of the physical objects has the change of its load. It is represented in case of the steam generator as a change or switch between devices, which are consuming the steam. Thus, the simulation studies over the control quality ratios were conducted. For this purpose, an integral quality factor, defined by the formula 6 was chosen. Factor called Integral Square Error (ISE) has a very pragmatic meaning. It represents the energy, which is lost at a particular type of control.

$$
I=\int_{0}^{\infty} e^{2}(t) d t
$$

After all of this assumption, the simulation studies were carried out. The results of those simulations for different operating points were presented in Table 2. According to those results, the best control quality is achieved around the assumed operating point. The integral quality factor reached the lowest value for this operating point.
Table 2. Value of ISE for different temperatures.

\begin{tabular}{|c|c|}
\hline Temperature $[\mathbf{C}]$ & ISE \\
\hline 110 & 4.766 \\
\hline 120 & 4.563 \\
\hline 130 & 4.415 \\
\hline 140 & 5.123 \\
\hline 150 & 5.877 \\
\hline 160 & 6.687 \\
\hline 170 & 7.549 \\
\hline 180 & 8.463 \\
\hline 190 & 9.439 \\
\hline 200 & 10.460 \\
\hline 210 & 11.533 \\
\hline
\end{tabular}

For higher temperatures selected settings are therefore worse, but in contrast to the hysteresis regulation type, they do not cause oscillations and instability. The output error is eliminated and the overshoot, due to reduced integral gain, is small. For other temperature operating point, only the control time may be increased. Adaptive control can be applied in order to overcome this disadvantage. However, it is much more computationally expensive than the classic PID algorithm.

Calculated tuned parameters were implemented into the PLC controller, and the results of the experiment on the real system are shown in figure 13.
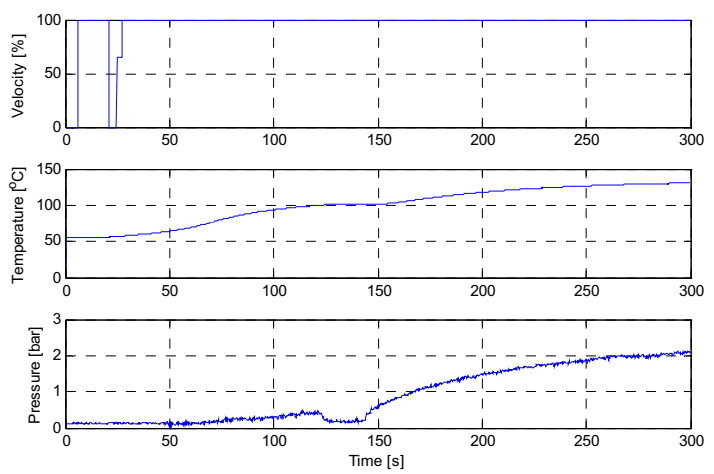

Figure 13. Control results for a real steam generator with tuned PID controller.

\section{Characterization of the generator operation}

The modified steam generator according described procedure was tested. The control and data acquisition was performed using dedicated software. The main panel is presented in figure. 14 .

The blocks responsible for control of the circulating pump, the burner ignitron system, the fuel pump/air fan frequency inverter are visible. Users are allowed to set working temperature and pressure of the medium and limits of those parameters. The control lights indicate status of the pump and burner. The variation of the parameters may be observed in real time on the graphs. Exemplary results of the generator operation are presented in figure. 15. 


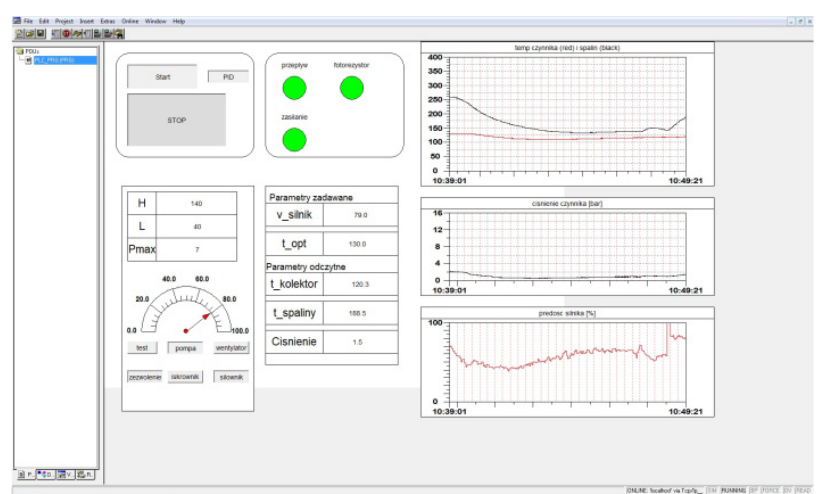

Figure 14. Main panel of the dedicated control software.

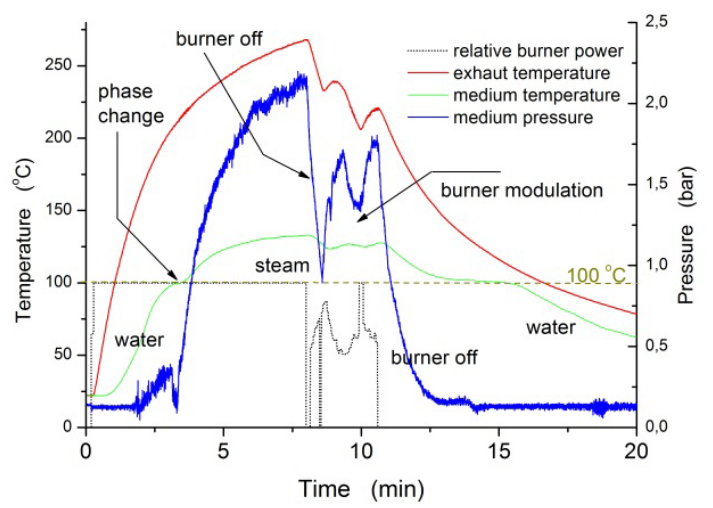

Figure 15. Main characteristics of the steam generator operation.

The test was performed for the partially open circuit steam, via the steam bus was directed to container with 100 liters volume. Part of the steam condensed and the rest was released to the atmosphere. This arrangement resulted relatively low steam pressure during the test - maximally ca. 2 bars.

The initial phase of the test is clearly visible the water temperature increase from the ambient temperature to $100 \mathrm{oC}$ - to beginning of the evaporation. This phase took about 3 minutes. The inverter works in this period with nominal frequency (100\%). Then the evaporation starts and the steam temperature increase till reaching setting value. The small temperature fluctuations around setting value are visible.

\section{Conclusions}

It was show that adaptations of simple and currently obsolete military steam generators for purpose of small, home cogeneration systems are possible. The possibility of creation of a full control model of such units allows for further, deeper modifications - e.g. burner replacement or modification of the combustion chamber. In such a case a repetition of the whole tuning procedure should be performed, what is related with different dynamics of burners and heat generation area.

Additionally, this paper presents the procedure of the fine-tuning of the PID controller parameters. A concise procedure of the offline method was presented.
The conditions of the identification procedure were given, and subsequently the reverse model of the real object was delivered. Designed numerical model was used to determine the optimal value of the controller parameters. This paper presents also discussion about the optimization of selected settings for other operating points. For this purpose, the integral quality factors were used.

Disadvantages of the most frequently used type of control system, which is the relay controller, are also discussed in the paper. Some disadvantages of classical continuous control using PID controller were also indicated. Those can be eliminated using its modification, called adaptive control. It allows to optimum process control in the full range of operational conditions, but unfortunately it is also much more computationally complex. This algorithm may therefore require increasing a memory of computing unit. However, in further development of this project it will be implemented.

\section{Acknowledgements}

The works have been conducted in collaboration between Dom Project Sp. z o.o. and AGH UST in Krakow (under statutory activities of the Faculty of Energy and Fuels "Studies concerning the conditions of sustainable energy development"). Purchase of the steam generator and its modifications were financed by Dom Project Sp. $\mathrm{Z}$ o.o. Components, used in the tests, were purchased with the funds of the "BioORC: Construction of cogeneration system with small a medium size biomass boiler" project.

\section{References}

1. K. Sornek, M. Filipowicz, K. Izdebski. Journal of Civil Engineering, Environment and Architecture, 62, 2 (2015)

2. K. Sornek, M. Filipowicz, M. Szubel, E. Bożek, K. Izdebski, MATEC Web of Conferences, 18 (2014)

3. R. Wróblewski, Energia Gigawat, 10-11 (2014)

4. EU Energy, Transport and GHG Emissions Trends to 2050, Reference Scenario 2013

5. K. Sornek, M. Szubel, W. Goryl, E. Bożek, M. Filipowicz, Przemysł Chemiczny, 93, 12 2014)

6. Y. Li, K.H. Ang, G.C.Y. Chong, Control Systems, IEEE, 26, 1 (2006)

7. Y. Li, K.H. Ang, G.C.Y. Chong, IEEE Control Systems Magazine, 26, 1 (2006)

8. J. Wołoszyn, A. Gołaś, Energy Conversion and Management, 87 (2014)

9. J. Kwasniewki, I. Dominik, K. Lalik, Journal Of Vibroengineering, 14 (2012)

10. I. Dominik, Solid State Phenomena, 164 (2010) 